\title{
Tensiones en el abordaje de la salud bucal en Colombia: caso ENSAB IV*
}

\author{
Tensions in the Oral Health Approach in Colombia: Case ENSAB IV
}

Tensões na abordagem da saúde bucal na Colômbia: caso ENSAB IV

Fecha de recepción: 18-09-2018 I Fecha de aceptación: 18-02-2019

\section{Fredy Fabián PARdo Romero}

Universidad Nacional de Colombia, Bogotá, Colombia. ffpardor@unal.edu.co. https://orcid.org/0000-0001-7698-8906

\section{LUIS JORGE HERNÁNDEZ FLÓREZ}

Universidad Nacional de Colombia, Bogotá, Colombia. luishern@uniandes.edu.co, ljhernandezf@unal.edu.co. https://orcid.org/0000-0001-7582-1557

\section{Lorena AleXandra Maldonado Maldonado}

Universidad Nacional de Colombia, Bogotá, Colombia. lamaldonadom@unal.edu.co. https://orcid.org/0000-0002-0997-9804

*Artículo de investigación original 
Correspondencia: ffpardor@unal.edu.co; luishern@uniandes.edu.co; ljhernandezf@unal.edu.co; lamaldonadom@unal.edu.co

doi: https://doi.org/10.11144/Javeriana.uo38-80.tasb

Cómo citar: Pardo Romero FF, Hernández Flórez LJ, Maldonado Maldonado LA. Tensiones en el abordaje de la salud bucal en Colombia: caso ENSAB IV. Univ Odontol. 2019 ene-jun;38(80). https://doi.org/10.11144/Javeriana.uo38-80.tasb

\section{RESUMEN}

Antecedentes: El Cuarto Estudio Nacional de Salud Bucal (ENSAB IV) representa un hito en los estudios nacionales de salud en Colombia debido a que se plantea desde un enfoque teórico alternativo y crítico llamado Determinación Social del Proceso Salud Enfermedad (DSSE). Sin embargo, operacionalizar ese enfoque teórico implicó desafíos importantes y generó algunas tensiones que limitaron la perspectiva crítica y transformadora que orientaba este estudio. Objetivo: mostrar las principales tensiones y desafíos epistemológicos y prácticos en el proceso de diseño y ejecución de la ENSAB IV. Métodos: Se realizó un estudio de caso triangulando información de fuentes primarias y secundarias. Se realizaron entrevistas semiestructuradas a actores clave que participaron en las diferentes fases del estudio y se hizo un análisis de contenido del protocolo y el informe final del estudio. Resultados: Las principales tensiones y desafíos evidenciados fueron el cambio de instituciones y actores responsables de las fases de diseño y ejecución; la no apropiación del enfoque teórico - metodológico en los resultados y 
recomendaciones finales y la falta de visibilización y articulación del componente cualitativo dentro del estudio. Conclusión: La fragmentación del proceso de diseño y ejecución del estudio y el cambio de actores responsables de cada fase, generó dificultades en la operacionalización del enfoque teórico-metodológico propuesto en el diseño del ENSAB IV, tanto por la poca orientación metodológica del enfoque en sí mismo, como por la resistencia institucional a mostrar resultados sanitarios desde perspectivas críticas.

\section{Palabras clave}

Colombia; determinantes sociales de la salud; estudio de caso; estudios epidemiológicos; investigación cualitativa

\section{Áreas temáticas}

epidemiología; epidemiología social; odontología; política de salud; política de salud bucal; salud bucal; salud colectiva; salud pública

\section{ABSTRACT}

Background: The Fourth National Study of Oral Health (ENSAB IV) represents a milestone in national health studies in Colombia because it arises from an alternative and critical theoretical approach called Social determination of the health disease process (DSSE). However, operationalizing this theoretical approach involved important challenges and generated some tensions that limited the critical and transformative perspective that guided this study. Purpose: To show the main tensions and challenges epistemological and practical in the process of design 
and execution of the ENSAB IV. Methods: A case study was conducted triangulating information from primary and secondary sources. Semi-structured interviews were conducted to key stakeholders who participated in the different phases of the study and a content analysis of the protocol and the final study report was made. Results: The main tensions and challenges highlighted were the change of institutions and actors responsible for the design and execution phases; the non-appropriation of the theoretical-methodological approach in the final results and recommendations and the lack of visibility and articulation of the qualitative component within the study. Conclusion: The fragmentation of the design process and implementation of the study and the change of actors responsible for each phase, created difficulties for the operationalization of the theoretical methodological approach proposed in the design of the ENSAB IV, both by the little methodological guidance of the approach in itself, as by the institutional resistance to show health outcomes from critical perspectives.

\section{Keywords}

case studies; Colombia; epidemiological studies; qualitative research; social determinants of health

\section{Thematic fields}

collective health; dentistry; epidemiology; health policy; oral health; oral health policy; public health; social epidemiology

\section{RESUMO}


Antecedentes: O quarto Estudo Nacional de Saúde Bucal (ENSAB IV) da um marco nos estudos nacionais de saúde na Colômbia porque surge a partir de uma abordagem alternativa e teórica crítica chamado Determinação Social do Processo de Doença-Saúde (DSSE). Embora, a operacionalização dessa abordagem teórica implicou desafios significativos e gerou algumas tensões que limitaram a perspectiva crítica e transformadora que orientou nosso estudo. Objetivo: avaliar as principais tensões e desafios epistemológicos e práticos no processo de concepção e execução da ENSAB IV. Métodos: Estudo de caso com triangulação de informações obtidas de fontes primárias e secundárias. Se realizaram entrevistas semi-estruturadas com atores-chave que tido participado das diferentes fases do estudo. Finalmente, foi feita uma análise do conteúdo do protocolo e do relatório final do estudo. Resultados: As principais tensões e desafios evidenciados foram, a mudança de instituições e atores responsáveis pelas fases de projeto e execução, a carência da apropriação da abordagem teórico - metodológica nos resultados finais e recomendações, e a falta da visibilidade e articulação do componente qualitativo dentro do estudo. Conclusão: A fragmentação do processo de desenho e execução do estudo, assim como a mudança dos atores responsáveis por cada fase, geraram dificuldades na operacionalização da abordagem teóricometodológica proposta no projeto da ENSAB IV. Isso pela pouca orientação metodológica, como pela resistência institucional para mostrar resultados de saúde desde perspectivas críticas.

\section{Palavras-chave}

Colômbia; determinantes sociais da saúde; estudos de caso; estudos epidemiológicos; pesquisa qualitativa

\section{Áreas temáticas}


epidemiologia; epidemiologia social; odontologia; política da saúde; política de saúde bucal; saúde bucal; saúde coletiva; saúde pública

\section{INTRODUCCIÓN}

El Cuarto Estudio Nacional de Salud Bucal (ENSAB IV) llevado a cabo entre los años 2009 y 2014 es uno de los ejercicios investigativos más ambiciosos y completos en el área de la salud que ha financiado el Estado colombiano en las últimas décadas; no solo por el gran despliegue de recursos humanos, técnicos y financieros alrededor de este estudio, sino por el novedoso marco conceptual y metodológico que asumió y planteo un desafío innovador en los Estudios Nacionales de Salud al asumir la propuesta desde el enfoque epidemiológico de la "Determinación Social del Proceso Salud Enfermedad" $(1,2)$.

El ENSAB IV, se llevó a cabo en dos grandes fases; en la primera fase se elaboró el diseño muestral, el protocolo y el estudio de costos y en la segunda fase se ejecuta dicho protocolo. La primera fase la desarrollo la Facultad Nacional de Salud Pública de la Universidad de Antioquia entre los años 2009 y 2010; y la fase de ejecución se realizó entre los años 2012 y 2014, y estuvo a cargo de la unión temporal Centro de Proyectos para el Desarrollo (CENDEX) de la Pontificia Universidad Javeriana y Sistemas Especializados de Información SEI S.A. La separación del Estudio en dos fases a cargo de diferentes actores e instituciones, generó una serie de desafíos adicionales para los involucrados en su diseño y ejecución, lo que derivó en la evidencia de una serie de tensiones entre los actores participantes y las formas de hacer convencionales de las 
instituciones; observándose además diferencias entre el análisis propuesto en la fase de diseño y la ejecución $(1,3)$.

En el momento en que se diseñó el protocolo del ENSAB IV, estaba vigente el Plan Nacional de Salud Pública 2007-2010 (PNSP) que asumía una visión de la salud muy centrada en la enfermedad y sus factores causales o de riesgo. En este plan, la salud oral era una de las 10 prioridades nacionales en salud, propuestas en ese momento; los enfoques teóricos que sustentaban este plan eran: el poblacional, el enfoque de determinantes propuestos por Lalonde y la gestión social del riesgo; todos estos enfoques compartían un núcleo epistemológico positivista particularmente el del riesgo en salud y unas apuestas en el hacer reduccionistas orientadas a la intervención y abordaje de aquellos factores causantes de las enfermedades que podían ser modificados, haciendo un énfasis particular en los hábitos y comportamientos de las personas (4).

Pese a las orientaciones conceptuales del PNSP, las discusiones teóricas en materia de salud en el país, particularmente en las esferas académicas, estaban muy activas debido, por un lado a la creación en 2005 de la Comisión de Determinantes Sociales de la Salud (CDSS) por parte de la Organización Mundial de la Salud (OMS), con una propuesta conceptual que superaba el Modelo de Determinantes de Lalonde; y por otro lado, a la sentencia T-760 emitida por la Corte Constitucional en el 2008 que declara la salud en Colombia como un derecho fundamental y no sólo un servicio público a cargo del Estado, como había quedado en la Constitución de 1991(5). En este contexto coyuntural de gran reflexión y deliberación académica en que se apuesta a desarrollar una visión amplia del derecho a la salud y a visibilizar lo social como determinante 
esencial en la configuración de los perfiles de salud y enfermedad tanto individuales como colectivos; es que se diseña el protocolo del ENSAB IV $(1,6)$.

La Institución responsable de esta primera fase del Estudio en 2009, había sido una de las líderes en las discusiones sobre Determinantes Sociales de la Salud (DSS) y los diferentes enfoques teóricos desarrollados sobre el tema, en este sentido, aunque se reconocían los avances del modelo propuesto por la OMS particularmente en la recuperación de la Atención Primaria en Salud (APS) y en la superación de la noción limitada de los cuatro factores determinantes de la salud propuestos por Lalonde; opto por asumir en el diseño del ENSAB IV el modelo de Determinación Social del Proceso Salud Enfermedad (DSSE), propuesto por la Medicina Social y Salud Colectiva Latinoamericana (MSSCL), fundamentado en el materialismo histórico y las teorías críticas provenientes de las ciencias sociales (7).

El modelo elegido como referente teórico del protocolo, planteaba una forma distinta de entender el proceso salud - enfermedad bucal en la población colombiana, concretamente la propuesta fue superar la noción biologicista de la salud bucal como la ausencia de patologías orales observables empíricamente en el nivel individual, y abordarla como un proceso histórico - social, multidimensional, dinámico y complejo (1). Esto implicaba retos importantes sobre todo en lo operativo, ya que no existía una experiencia previa de aplicación del modelo de DSSE en estudios poblacionales de esta envergadura.

El plan de análisis propuesto en el diseño del ENSAB IV, pretendía entonces "trascender la simple medición del daño al sistema estomatognático" para dar cuenta de los procesos sociales que 
explicaran el proceso salud enfermedad bucal en el país, visibilizando las formas diferenciales en que se configuran y se expresan los perfiles epidemiológicos bucales según la inserción social por clase, género y etnia (1). Adicionalmente, se propuso evaluar indirectamente el impacto que ha tenido el actual Sistema de Salud, en la salud bucal de los colombianos (1).

Para lograr esta aproximación, se planteó un estudio con un componente cualitativo y otro cuantitativo en una muestra representativa de la población colombiana, que permitiera abarcar y analizar varios planos de interpretación de las relaciones que se dan entre la dimensión material y la dimensión subjetiva de la realidad social de la salud bucal y no solo describir el comportamiento de los indicadores de morbilidad bucal y sus posibles factores causales (1). Se propuso la clase social como categoría central en el análisis de resultados y se definieron tres niveles de análisis: Análisis de inequidades en salud bucal; Análisis de desigualdades sociales en salud bucal y Análisis descriptivo de la frecuencia y distribución de los problemas de salud bucal según edad, zona de procedencia, sexo y afiliación a la seguridad social. El análisis de inequidades buscaba evidenciar cómo las estructuras sociales, económicas, políticas y jurídicas que prevalecen en el país producen y reproducen sistemáticamente injusticias sanitarias y cuáles eran los grupos poblacionales más vulnerables según su inserción de clase, género y etnia. Por su parte, en el análisis de desigualdades, entendidas éstas como las diferencias en salud bucal producidas por las inequidades sociales, que son empíricamente evidenciables; se proponía un ejercicio comparativo de los principales indicadores de la salud bucal observable, del acceso a servicios sanitarios y de elementos subjetivos, en función tanto de los diferentes perfiles de clase (modos de vida) como del ciclo de vida y otras condiciones de interés como el desplazamiento y la discapacidad (1). Finalmente, el análisis descriptivo propuesto en el tercer nivel, tenía por objeto calcular los 
indicadores que permitieran comparar los resultados con el anterior estudio Nacional Salud Bucal (ENSAB III), además también con indicadores internacionales y analizar tendencias en términos estadísticos (8).

La información necesaria para realizar todos los análisis propuestos en el protocolo se obtendría de una evaluación clínica y la aplicación de encuestas estructuradas a todas las personas de la muestra definida y de la realización de entrevistas a profundidad a una sub-muestra de 48 personas que fueran representativas de la diversidad de clase social, género, etnia y zonas de menor y mayor prevalencia de enfermedad bucal. Visibilizar y comprender las dinámicas diferenciales del proceso salud - enfermedad bucal en la población colombiana, en diferentes niveles concatenados, sería fundamental para orientar transformaciones profundas en lo social, lo político y en las practicas odontológicas en función de la equidad y el bienestar (1).

Una vez se aprueba el protocolo por parte del entonces Ministerio de Protección Social, se abre una nueva licitación para su ejecución. Esta segunda fase queda a cargo de la unión temporal Centro de proyectos para el desarrollo (CENDEX) de la Pontificia Universidad Javeriana y Sistemas Especializados de Información (SEI), que inician el trabajo encomendado en el año 2012. Materializar la propuesta plasmada en el protocolo, implico varios desafíos y dificultades particularmente en lo operacional, que desencadenaron en cambios tanto de forma como de fondo del diseño inicial y tensiones evidenciadas por los actores involucrados $(1,3)$.

A nivel de políticas públicas, para el año 2012 había entrado en vigencia el Plan Decenal de Salud Pública 2012-2021 (PDSP), que planteo como objetivos principales: reducir las inequidades en 
salud, mejorar condiciones de vida y salud y no permitir la morbi-mortalidad y discapacidad evitable. Para cumplir con estos objetivos, este nuevo plan incorporó múltiples enfoques teóricos como el enfoque de derechos, el de los determinantes sociales propuesto por la OMS, el enfoque diferencial por ciclo vital, género y etnia y el enfoque poblacional. En este PDSP, la salud bucal dejo de ser una prioridad explicita y se incorporó a una de las nuevas dimensiones prioritarias denominada "Vida saludable y condiciones no transmisibles"(9). Pese a lo amplio de los enfoques teóricos propuestos, las estrategias concretas que plantea el PDSP para abordar la salud bucal, siguen centradas en la identificación y control de factores de riesgo modificables sobre todo en la esfera individual, el autocuidado y la atención clínica (9).

El objetivo de este artículo original de investigación de tipo cualitativo basado en fuentes primarias y secundarias de información es aproximarse y visibilizar las principales tensiones y desafíos generados debido al enfoque teórico metodológico utilizado y a las diferencias observadas entre la propuesta inicial y lo finalmente ejecutado.

\section{MATERIALES Y MÉTODOS}

Este trabajo se desarrolló como un estudio de caso, con base a la relectura del informe final de Cuarto Estudio Nacional de Salud Bucal (ENSAB IV) y del protocolo inicial diseñado por la Universidad de Antioquia (UdeA); la información se obtuvo de fuentes primarias y secundarias mediante la revisión de los documentos mencionados anteriormente y la implementación de una entrevista semiestructurada realizada a actores clave que participaron en la fase de diseño inicial, en la fase de ejecución, del comité técnico y supervisor del Ministerio de Salud y Protección social 
y del grupo de asesores participantes; a través de unas dimensiones de análisis con el objetivo de identificar las principales tensiones generadas por el enfoque teórico metodológico abordado y la fractura del estudio en dos fases (diseño y ejecución) a cargo de actores e instituciones diferentes.

Se realizaron entrevistas semiestructuradas a partir de preguntas orientadoras y en profundidad de acuerdo a categorías de interés para el estudio, a algunos de los participantes en el diseño inicial y en la ejecución del estudio; además de algunos de los integrantes del comité técnico y supervisor del Ministerio de salud y protección social y del grupo de asesores del estudio.

Se realizó una primera entrevista semiestructurada mediante preguntas orientadoras construidas usando criterios preliminares; a diferentes actores participantes con una duración promedio de 120 minutos, posteriormente fue realizada la transcripción de cada una de las entrevistas realizando una lectura y relectura de cada una de ellas. Una vez finalizado este proceso se programó una segunda sesión con una duración aproximada promedio de 40 minutos con algunos de los actores participantes para profundizar, corregir o aclarar algunos de los aspectos de esta. Los actores participantes fueron divididos en cuatro grupos de acuerdo a las funciones realizadas en el estudio: Integrantes del equipo que participo en el diseño del protocolo (3); del equipo que participo en la ejecución del protocolo (6); del equipo del Ministerio de salud y Protección Social (técnico y supervisor) (2); e integrantes del equipo asesor (2).

En total se contó con la participación de trece personas de los equipos mencionados anteriormente: seis participantes del equipo que realizó la ejecución del protocolo; tres del equipo que realizo el diseño inicial; dos del equipo del Ministerio de Salud y Protección Social y dos del equipo asesor del estudio. Los datos obtenidos fueron procesados y sistematizados mediante el software N-vivo 
12 plus realizando un proceso continuo de comprensión, síntesis, teorización y recontextualización.

\section{Consideraciones éticas de la investigación}

La presente investigación no incluyó ninguna intervención sobre seres humanos que implicase algún riesgo físico, psicológico o moral para los /las participantes. Esta se acoge a la resolución 008430 de 1993 del Ministerio de Salud de la República de Colombia; "por la cual se establecen las normas científicas, técnicas y administrativas para la investigación en salud"(10). De acuerdo a esta resolución la presente investigación se cataloga sin riesgo para los/las participantes. La información suministrada por los/las participantes se usó única y exclusivamente para el logro de los objetivos de la investigación. Además se aseguró la confidencialidad de la información suministrada y el anonimato de los/las participantes (10).

De acuerdo al principio de autonomía se solicitó a los/las participantes diligenciar un consentimiento informado en el cual se le suministró una información adecuada y suficiente acerca del estudio y sobre la posibilidad de abandonar la entrevista en cualquier momento de acuerdo a su conveniencia (Figura 1). Finalmente, en todo momento se procedió de acuerdo a los principios éticos de autonomía, beneficencia, no maleficencia y justicia. 
FIGURA 1

CONSENTIMIENTO INFORMADO

UNIVERSIDAD NACIONAL DE COLOMBIA SEDE BOGOTÁ

DOCTORADO INTERFACULTADES EN SALUD PÚBLICA

"Modelos epidemiológicos que han orientado los Estudios Nacionales de Morbilidad oral - Salud Bucal en Colombia"

Mediante este formato de "consentimiento informado", confirmo que he sido invitado/da a participar en esta investigación; de la cual conozco sus objetivos y su justificación. Además, he sido informado/da de los siguientes aspectos:

1) Me realizaran varias preguntas relacionadas con la investigación y la información que suministre será de carácter confidencial.

2) Puedo desistir de la entrevista durante cualquier momento de la realización de la misma.

3) Las preguntas, que surjan de mi parte antes, durante o después de la entrevista serán contestadas en el momento que lo solicite.

4) Se me ha informado que esta investigación no generara ningún tipo de riesgo para mi persona.

5) Adicionalmente, las actividades realizadas no me generaran ningún costo.

6) Además, he recibido la información correspondiente al nombre del investigador responsable "Fredy Fabián Pardo Romero" y los datos de contacto N. ${ }^{\circ}$ teléfono celular 3008284870 y correo electrónico: ffpardor@unal.edu.co.

Por lo anterior acepto participar voluntariamente en esta investigación, de igual forma entiendo que por mi participación en este estudio no recibiré retribución alguna; sin embargo, si lo deseo expresamente podré recibir una copia del informe final una vez concluya este estudio.

En acuerdo con los anteriores aspectos, procedo a firmar a los días, del mes del año en la ciudad de

Participante:

Testigo:

Nombre Nombre

Firma Firma

Cédula N. ${ }^{\circ}$ Cédula N. ${ }^{\circ}$

Investigador:

Nombre

Firma Cédula N. ${ }^{\circ}$

Este trabajo muestra resultados parciales de la tesis doctoral "Modelos epidemiológicos que han orientado los Estudios Nacionales de Morbilidad Oral - Salud Bucal”; investigación aprobada mediante Acta de Evaluación N. ${ }^{\circ}$ 013-127-15 del Comité de Ética de Investigación de la Facultad de Medicina de la Universidad Nacional de Colombia con fecha 13 de Agosto de 2015 (Figura 2). 
FIGURA 2.

Comité de Ética, Facultad de Medicina. ACta de Evaluación
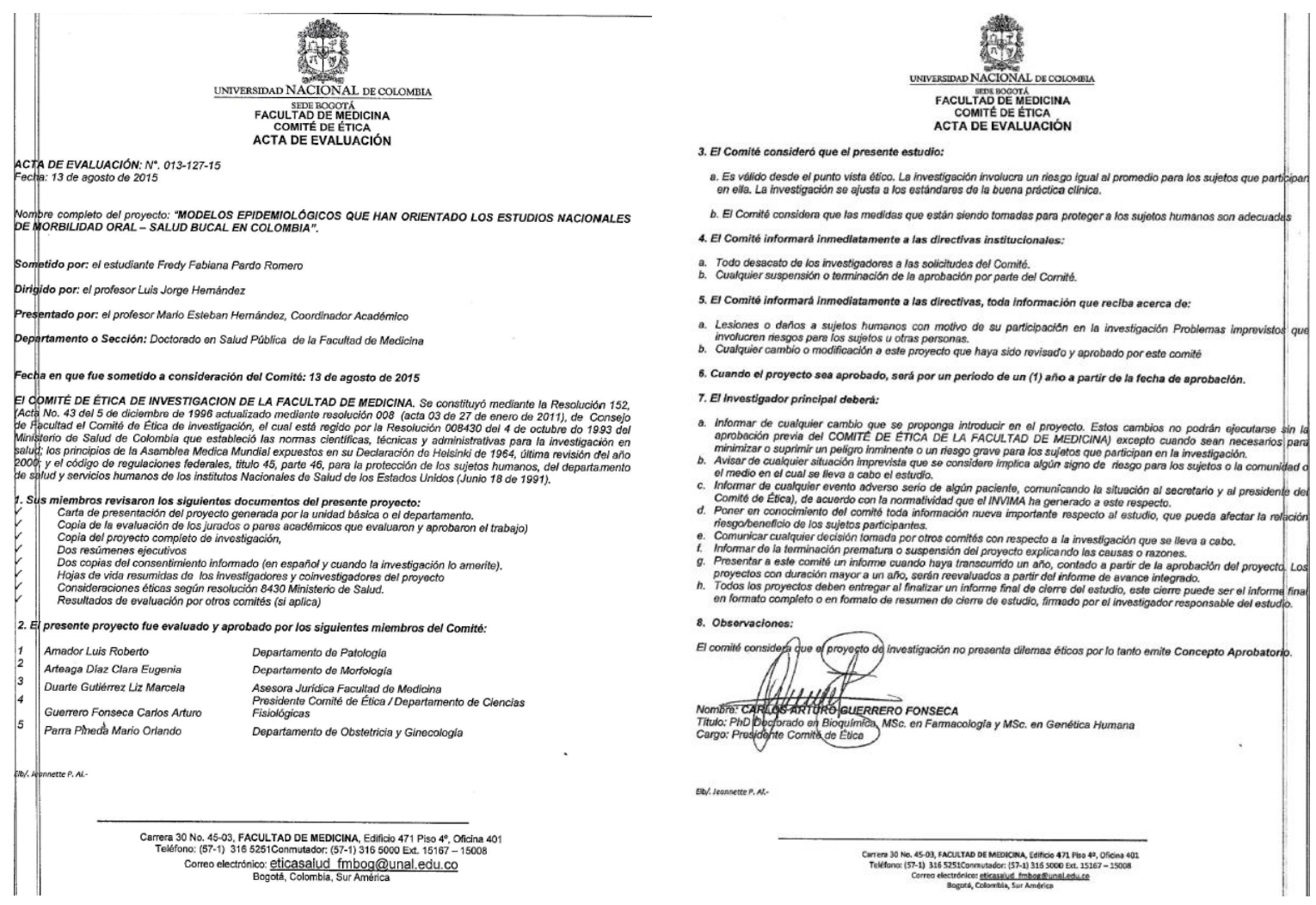

\section{RESULTADOS}

Al recabar información de fuentes primarias y secundarias de la información se evidencian una serie de tensiones y desafíos relacionadas con la apropiación y operacionalización del enfoque teórico metodológico propuesto; el desarrollo y desarticulación del componente cualitativo y con el hecho de fracturar el estudio en dos fases con actores e instituciones diferentes encargadas del desarrollo de cada una de ellas. Algunas de las principales tensiones evidenciadas de acuerdo a las narrativas de los actores participantes fueron: 


\section{Tensión y desafío percibido en la apropiación y operacionalización del enfoque teórico- metodológico por los equipos encargados del diseño y la ejecución del estudio}

Materializar la propuesta desde el enfoque epidemiológico de la determinación social de la salud plasmada en el protocolo inicial, implico varios desafíos y dificultades particularmente en lo operacional, que desencadenaron tensiones manifestadas por varios actores que participaron en cada uno de los grupos mencionados anteriormente; por cuanto el nivel de apropiación del abordaje teórico metodológico fue diferente de acuerdo a lo referido por los participantes en cada de las fases del estudio como se evidencia en las narraciones de algunos de ellos.

Las narrativas presentadas a continuación corresponden a las percepciones de algunos de los participantes en la fase del diseño inicial del estudio:

...llegue a la conclusión de que digamos que ese componente de la Determinación Social, se debía haber como trabajado más, que es muy iluso pensar que en un Estudio Nacional de estas magnitudes se puedan hacer esos abordajes que requieren un componente de esos y que entiendo absolutamente todo lo que me comento el equipo ejecutor de las dificultades que tuvieron para procesar ese componente del estudio las entiendo perfectamente... (Integrante del equipo que participó en la fase inicial de diseño del protocolo del estudio).

...pero de todas maneras si se sintió esa tensión y esas discusiones con los que venían de Bogotá y eso se discutía pues, como se iban hacer las preguntas; esto generó mucha dificultad y además vino el experto y ayudó a hacer eso y después sobre todo a mí me parece que hubo más dificultad cuando los que ya fueron a ejecutar el estudio tomaron esta Determinación Social y yo creo que para ellos fue una cosa muy... pues como lo 
planteamos nosotros yo creo que fue muy difícil para ellos acomodarse a eso que no sé, aunque el experto también fue allá, ayudó en el análisis de eso, pero me parece que esas son tensiones que se generan por qué la gente no internaliza muy fácil esa Determinación Social de la Salud... (Integrante del equipo que participó en la fase inicial de diseño del protocolo del estudio).

Esta dificultad en la apropiación y operacionalización del enfoque se confirma en la narrativa de algunos integrantes del equipo que realizo la ejecución del protocolo quienes mencionan el desafío que significo realizar la adaptación de los instrumentos a la realidad de la sociedad colombiana:

En términos generales, el primer tema es que era un diseño, una idea de hacerlo desde una aproximación de la Determinación Social, pero esa aproximación desde la determinación social había que volverla operativa... El Ministerio pensaba que era lo mismo Determinación Social y Determinantes Sociales y ellos su enfoque son Determinantes Sociales entonces, cuando se encuentran con este estudio, ellos dicen oiga ese no es el enfoque que nosotros queremos; entonces nosotros le dijimos, pero ese es el enfoque que usted contrato y no me puedo devolver todo el proceso que hubo de diseño. Entonces nos vamos con el enfoque de la Determinación social; y en ese sentido lo que se hizo con todo el equipo de asesores fue de tratarlo de hacerlo operativo... (Integrante del equipo que participó en fase de ejecución del protocolo del estudio).

Deberían mirarse muchos otros casos, siento que todavía hay cosas que decir y siento que en el marco de eso, faltaban muchas claridades y faltaba aterrizarlo a las condiciones de la población colombiana, porque con la categoría de clase social a veces uno no identifica, 
incluso saliéndonos de los casos y yéndonos al análisis de la Determinación Social, solamente la mitad de la población quedaba asignada a una fracción social... pero debió haberse hecho una prueba piloto, nos mandaron a probar el operativo de campo nunca a los instrumentos, porque no era nuestra función, entonces para mí faltó una prueba piloto que nos permitiera ajustar, una prueba piloto para identificar las categorías (Integrante del equipo que participó en fase de ejecución del estudio).

Para algunos de los participantes del equipo de la institución que contrato el estudio, asumir el desafío de la operacionalización del enfoque teórico metodológico de la Determinación Social significo incluso cuestionar el desarrollo y alcance del instrumento propuesto para la recolección de la información:

...ya nos habían salido canitas pero muchas nos la saco determinantes y por ejemplo a los integrantes del equipo que realizaron la ejecución del estudio sí que le saco canas porque como concepto y enfoque ideológico está clarísimo y están claras las diferencias frente a determinantes sociales y determinación social desde lo ideológico y lo conceptual; desde lo operativo eso no tiene nada, nada y ... esa es una debilidad de quienes hicieron el protocolo porque están inmersos en lo conceptual pero no se imaginaron como era lo logístico o si se lo imaginaron no sabían cómo cogerlo (Integrante del equipo del Ministerio de Salud y Protección social).

...eso lo hace otra universidad y no se va hacia el enfoque de Determinación Social, porque digamos también fue un problema meter esa encuesta a Determinación Social, en los estudios que hicimos nosotros en las revisiones que hicimos nosotros, incluso con el 
experto le dijimos mire, esto a nivel de encuesta poblacional como se hace, entonces él nos dice "yo no sé, yo no lo he hecho", o sea nosotros le hicimos la tarea a ..., le hicimos la tarea de aplicar ese modelo, cómo se comporta en ese modelo a una encuesta poblacional de una magnitud y una complejidad mayor... (Integrante del equipo del Ministerio de Salud y Protección social)

En cambio, para este participante del equipo asesor del diseño inicial el problema no es el enfoque teórico metodológico sino el conocimiento y apropiación por parte del equipo profesional encargado de la ejecución:

...mi perspectiva cuando lo escucho es que quienes lo dicen, no entienden o tienen limitaciones para el entendimiento de la perspectiva entonces aducen un problema de dificultad de operacionalización para de alguna manera desechar el enfoque eso es digamos lo que yo he visto en términos generales para darte un ejemplo especifico porque desconozco la discusión que tuvo la institución encargada de la ejecución y demás, pero en otros proyectos en otras experiencias entre investigadores siento que hay, un pretexto para desechar el enfoque desde esta perspectiva en el que se aduce que hay problemas de operacionalización y al haber problemas de operacionalización poder tener un elemento que justifique buscar otro enfoque, alguna cosa así y lo que veo más bien son dificultades, limitaciones en la apropiación del enfoque...

Estas experiencias narradas por actores con diferentes funciones dentro del estudio permiten aproximarse al desafío que significo apropiar y operacionalizar el enfoque teórico metodológico 
propuesto en un estudio poblacional con una muestra importante y de acuerdo a las complejidades del contexto social colombiano.

\section{Tensión entre enfoques teórico - metodológicos: la postura oficial Vs la Determinación Social de la Salud}

A pesar de que tanto en el objetivo general del protocolo del estudio como del informe final de la ejecución del protocolo; se menciona como eje principal del estudio "Caracterizar las condiciones de salud bucal de la población colombiana y aproximarse comprensivamente a los procesos de Determinación Social de la salud y su componente bucal"; sin embargo se evidencia en el informe final publicado en 2015 como en el análisis de los resultados se trataron de incorporar algunos referentes teóricos desde la postura oficial de los Determinantes Sociales de la Salud, quedándose sin embargo cortos en los desarrollos y análisis de los resultados como lo resaltan algunos de los actores participantes en el estudio (11):

Yo creo que el Ministerio no se ha dado el espacio para generar el debate alrededor de los Determinantes sociales versus la Determinación, yo creo que el Ministerio sigue la línea de los determinantes, pero lo ve como una relación todavía desde un enfoque de factores de riesgo, entonces consideran que los determinantes son factores de riesgo para el desarrollo de la enfermedad, siendo todavía muy patocéntrico; si entonces en este sentido parte de las discusiones que nosotros hicimos fueron, que es muy difícil cuando tu contratas a una firma para el diseño del estudio y a otra para su ejecución, porque el que ejecuta termina diciendo yo ejecuto lo que ustedes diseñaron, entonces ahí no hubo espacio para un debate conceptual, ahí sigue la línea de un enfoque patocéntrico donde yo determino que lo importante son las patologías bucales y no los determinantes que podrían estar 
subyacentes a las condiciones de salud bucal... (Integrante del equipo que participó en fase de ejecución del protocolo del estudio).

... en el primer libro se trata de hacer una descripción de la Determinación Social, pero se toman elementos de los Determinantes Sociales porque también era una necesidad del Ministerio de que se pudiera generalizar el enfoque de los Determinantes Sociales ya que ellos todos sus estudios poblacionales los hacen por ahí, entonces si tú me preguntas hay una mezcla de los dos elementos. Ahí hay una discusión teórica enorme; sin embargo, para algunos autores son complemento, para otros autores son divergentes estos enfoques, pero se trató de mantener los dos (Integrante del equipo que participó en fase de ejecución del protocolo del estudio).

De parte de este integrante del equipo técnico y supervisor de la institución que contrato el estudio, se ratifica una clara alineación por parte de del Ministerio de Salud con la postura oficial OMS de los Determinantes Sociales de la Salud y Protección Social.

El ministerio y el país venían hablando de Determinantes Sociales pero no los había acogido de manera oficial, digámoslo así pero si estaba alineado con OMS por muchas razones, haber nosotros hacemos parte y financiamos OMS, somos un país que esta aliado con OMS y OMS se financia con los recursos que le dan todos los países, nosotros hacemos parte de OMS entonces digamos que lo conceptual es la construcción Estado y país muy alineado, porque se da ENSAB con Determinación Social? precisamente porque se lo gano la UdeA si se lo hubiera ganado una institución diferente de pronto no hubiera quedado con 
Determinación Social... (Integrante del equipo del Ministerio de Salud y Protección social).

A través de los documentos publicados con los resultados del estudio se observa que se toman elementos tanto del enfoque de factores de riesgo, de los Determinantes Sociales de la Salud- OMS y de la Determinación Social del proceso Salud - Enfermedad sin embargo no se evidencia un desarrollo en profundidad de los dos últimos, quedándose todavía los análisis muy anclados en la prevalencia de morbilidad oral de la población colombiana $(3,11)$.

\section{Tensión entre el diseño y la ejecución del componente cualitativo: Desarticulación del componente cualitativo}

En el protocolo estaban planteadas 48 entrevistas a profundidad de naturaleza hermenéutica, para interpretar las subjetividades y experiencias de los colombianos en torno a la salud bucal y articular esta información con los resultados de los datos cuantitativos para dar cuenta de los objetivos planteados; no obstante, en la ejecución del estudio se llevó a cabo un diseño cualitativo incrustado de 6 estudios de caso de carácter etnográfico que se consideraron independientes al ENSAB IV y secundarios en la medida en que no tenían representatividad numérica ni estaban orientados a responder al objetivo general de la investigación. En cuanto al plan de análisis, el planteamiento hecho en el protocolo de visibilizar los procesos estructurales generadores de inequidades para en seguida describir las desigualdades en salud bucal entre los diferentes grupos sociales de acuerdo a su inserción social y finalmente dar cuenta de la morbilidad evidenciable como resultado de un proceso de subsunción de la biología en lo social, implicaba una articulación y triangulación del componente cualitativo con el cuantitativo; no obstante en la ejecución del estudio, no se llevó a 
cabo dicha triangulación de técnicas, y la metodología cuantitativa se impuso como única forma de analizar la información recolectada $(1,11)$. A partir de análisis estadísticos descriptivos y correlacionales, se presentaron los resultados de los principales índices de morbilidad bucal y de desigualdades sociales en materia de salud bucal, asumiendo los elementos constitutivos de la determinación social como factores de riesgo o variables explicativas a-históricas, de la condición de salud bucal de los diferentes grupos sociales identificados. Tampoco se realizó una desagregación por clase social, género, etnia y acceso a servicios de salud de los modos de vida propuestos, como se planteó en el protocolo, generando dificultades en reconocer quienes concretamente tienen dichos modos de vida y que tipo de inequidades subyacentes pueden identificarse.

La desarticulación entre los componentes cualitativo y cuantitativo y la operacionalización de las dimensiones y categorías de análisis del modelo teórico de la DSSE sólo como variables estadísticas, limito las posibilidades de comprender las dinámicas socio-históricas de la salud bucal en el país, que por su naturaleza dialéctica, conflictual y compleja, no pueden ser captadas únicamente con modelos matemáticos lineales.

Varios de los actores participantes coinciden en la falta de profundidad en este componente y desarticulación del componente cuantitativo y cualitativo como lo evidencian sus narraciones:

Si en eso si falto un mayor esfuerzo creo yo, por eso nosotros lo habíamos planteado de otra manera, pero si se pueden poner a conversar mejor con los resultados de la encuesta aunque ellos hicieron el intento porque definen al principio que este caso corresponde a una persona que está en tal... si trataron de hacer una ubicación pues con el grupo social y 
otros aspectos de los modos de vida sobre todo, pero sin embargo esta parte si se podría mejorar (Integrante del equipo que participó en la fase inicial de diseño del protocolo del estudio).

Me acuerdo cuando estuvimos negociando la parte cualitativa, pues si mencionamos que dependiendo de la cantidad de recursos pues se podía hacer un estudio de mayor o de menos envergadura, y ahí pues hubo varias discusiones, por ejemplo, pues logramos hacer solo seis casos, hubiésemos querido que se hicieran muchas más cosas, muchos más estudios por regiones, pero no alcanzo debido a las limitaciones de los recursos, pero pues no era el componente central del ENSAB. Entonces pues si hubieran existido más recursos se hubiera podido hacer un estudio con mayor profundidad, con mayor trabajo de campo, o recolectar más casos...” (Integrante del equipo que participó en fase de ejecución del estudio).

Desde el protocolo se había planteado algo del enfoque cualitativo pero digamos que no somos... el país no está en capacidad de hacerlo las personas que nos ayudaron saben mucho de enfoque cualitativo y fueron las que nos ayudaron realmente ahí si les dejamos a ellos mucha de esa capacidad porque son los expertos y al menos se hizo alguna aproximación siquiera, seguramente sé que hay muchas maneras de haberlo abordado mejor o de haberlo abordado con mayor profundidad pero bueno hicimos una aproximación en ese cualitativo empieza a ver uno también esas otras necesidades que hay que abordar... (Integrante del equipo del Ministerio de Salud y Protección social). 
Además, varios de los participantes resaltan la desarticulación entre el componente cuantitativo y lo cualitativo e incluso los catalogan como dos estudios separados:

No, yo creo que nunca se intentó hacer algo mixto, yo creo que siempre se pensó en dos estudios, desde el protocolo, el protocolo decía un estudio cualitativo, pero si tú lo lees no está articulado, nunca, además es, digamos que estamos muy nuevos en esto, o sea en estudios mixtos; utilizamos herramientas cualitativas pero no estudios cualitativos anexados a una encuesta, siempre se sacó así, no sé si los expertos en estudios secundarios sacaron algo diferente, algo más integrador, desde mi concepto siempre se tomó como dos estudios, empezando por la muestra, ósea tú vas a ver la muestra de cualitativos no estaba amarrado a lo cuantitativo (Integrante del equipo del Ministerio de Salud y Protección social).

Porque creo, que fue un ejercicio que se hizo y nunca pretendió ir más allá de un ejercicio por la dificultad en un estudio nacional de una metodología de ese tipo, entonces lo que yo siempre entendí y no estuve involucrado en esa parte; es que era eso, un ejercicio para mirar si eso es viable en estudios nacionales y como se puede empezar a implementar entonces como yo tenía claro eso, no me sorprende que este desarticulado como efectivamente creo que esta, lo importante es que la gente que esté al frente de los nuevos estudios coja eso y haga ejercicios muchos más grandes en el próximo estudio nacional no solo de salud bucal, en los estudios nacionales de salud (Integrante del equipo que participó en fase de ejecución del protocolo del estudio). 
Se puede apreciar cómo, a partir de estas experiencias y a pesar de los esfuerzos realizados, no se logró establecer un dialogo entre los componentes cuantitativo y cualitativo y eso genero la percepción de estar ante dos estudios separados.

\section{Tensión al tener dos equipos diferentes uno para el diseño y otro para la ejecución del estudio}

La UdeA que había ganado la convocatoria para realizar la primera fase de diseño del Estudio, sin embargo, no fue la entidad seleccionada para realizar la ejecución del protocolo diseñado y eso genero una serie de discontinuidades e inconvenientes como lo narran varios de los actores participantes:

Pues uno siempre se queda con la sensación que cuando un equipo diseña y otro ejecuta ahí hay una brecha, una disonancia por llamarlo de alguna manera nosotros hubiéramos tomado otras decisiones, creo yo frente a esto aunque valoro el trabajo que hicieron la entidad encargada de la ejecución pero si hubo cambios que para nosotros posiblemente no se hubieran dado... frente al componente cualitativo que se había planteado en el marco del enfoque de la Determinación Social que también nosotros lo habíamos planteado ellos también hicieron unos cambios incluso cambiaron el tipo de estudio, nosotros teníamos más propuesto un enfoque hermenéutico y que íbamos a trabajar con determinados informantes clave por regiones si, y ellos pasaron a hacer un estudio de caso... (Integrante del equipo que participó en la fase inicial de diseño del protocolo del estudio).

A mí me parece que eso no es coherente yo creo que debe haber un equipo, conseguir un equipo que diseñe y ejecute; yo creo que esta brecha que se abre acá, yo me acuerdo de ellos, yo supe que ellos tuvieron que llamar por ejemplo al experto para entender esa cosa 
pues, entonces si el uno ya la armó y los otros bregando a entender lo que armaron otros eso me parece que se vuelve complejo... (Integrante del equipo que participó en la fase inicial de diseño del protocolo del estudio).

Otros actores participantes confirman las tensiones y desafíos generados a partir de tener dos instituciones diferentes para cada una de las fases del estudio.

Sin duda, pues claro el nivel de compromiso con la perspectiva teórica de convicción y de apropiación es distinto, no puedo decir que el trabajo de la institución encargada de la ejecución haya sido malo, no pero es distinto, no le puedes pedir a quien no lo diseño y a quien no tiene un nivel de apropiación y de convicción con una perspectiva teórica que haga un ejercicio con la misma profundidad y refinamiento a que lo haga quien lo diseño, sin duda... (Integrante del equipo asesor del estudio).

Eso de que alguien diseñe y otro implemente creo que produjo algunas discontinuidades y algunas complejidades de enlace y de desperdicio porque si ya se habían procesado una cantidad de cosas con este grupo bueno este grupo pasa su informe al otro y luego el otro implementa entonces habría que volver a ciertas cuestiones de... yo nunca entendí esa metodología de que alguien diseñe y otro ejecute... (Integrante del equipo asesor del estudio).

Sin duda para la institución encargada de la ejecución del protocolo el hecho de no haber realizado el diseño, implico un esfuerzo adicional, lo cual condujo a una serie de ajustes que se apartaron en cierto grado de la propuesta inicial. 


\section{DISCUSIÓN}

Una de las principales tensiones y desafíos identificados tiene que ver con los enfoques teóricometodológicos que sustentan tanto la comprensión del objeto salud bucal como las propuestas de acción que se derivan de cada postura epistemológica. Actualmente, por lo menos en la esfera académica, la concepción biologicista de la salud como ausencia de enfermedad se encuentra ampliamente revaluada. El nuevo conocimiento que se genera en salud cada vez más visibiliza la importancia de desarrollar y profundizar abordajes holísticos que permitan tener una visión integradora de la salud reconociendo su naturaleza compleja y multidimensional (6). Estos planteamientos holísticos han permeado algunos escenarios políticos importantes como la Organización Mundial de la Salud, logrando así que esta institución además de proponer una definición de salud que supera en buena medida el reduccionismo biológico, también haya visibilizado y posicionado políticamente la dimensión social de la salud creando en 2005 la Comisión de Determinantes Sociales encargada de estudiar las causas sociales de la falta de salud y las inequidades sanitarias evitables en contextos particulares, para así orientar políticas públicas integrales e integradas que respondan a las necesidades de las poblaciones $(12,13)$.

Si bien el enfoque teórico de DSS propuesto por la OMS basado en la epidemiología social europea se ha adoptado como postura oficial, no es la única propuesta conceptual que desarrolla la dimensión social de la salud. El movimiento de la Medicina Social y Salud Colectiva Latinoamericana (MSSCL), ha desarrollado una propuesta teórico política contra-hegemónica denominada la Determinación Social del proceso Salud Enfermedad (DSSE), que entiende la salud 
y la enfermedad como un continuum en el proceso vital humano que es dinámico, dialéctico, complejo, determinado social e históricamente, cuyo estudio y abordaje requiere la integración de múltiples ciencias y prácticas sociales y políticas transformadoras en diferentes niveles (14-16). Esta corriente latinoamericana, hace una importante crítica a los planteamientos de la OMS, por considerar que la definición que plantea de salud sigue siendo limitada y estática y que la noción de DSS como factores o causas a-históricas, invisibiliza los procesos estructurales de dominación (de clase, género y etnia) que producen y reproducen inequidades sanitarias, siendo así un modelo funcionalista que no plantea cambios profundos sino ajustes y modulaciones que terminan fragmentadas sin generar un impacto sostenido en la salud de los colectivos $(7,17)$.

El ENSAB IV es un ejemplo de cómo la institucionalidad sanitaria del Estado Colombiano se ha ido adaptando a los nuevos discursos en el abordaje de la salud, no es coincidencia que la propuesta teórico - metodológica planteada en el protocolo del estudio haya tenido una aceptación inicial por parte del entonces Ministerio de Protección Social, que posiblemente en su momento no identificó diferencias sustanciales entre la propuesta de DSS planteada por la OMS y la propuesta de DSSE planteada por la MSSCL, entre otras porque estas discusiones epistemológicas se daban más en el ámbito académico. Esta falta de claridad epistemológica desencadenaría una de las principales tensiones entre lo planteado en el protocolo y lo visibilizado en los resultados de la segunda fase del ENSAB IV, ya que las propuestas praxiológicas que derivan de cada enfoque son muy distintas. Por un lado los DSS al no visibilizar ni discutir las relaciones de poder y dominación de la sociedad, termina dando un mayor peso al estilo de vida (hábitos, comportamiento y exposición diferencial a factores de riesgo a-históricos) en la explicación de los niveles de salud, de allí que sus propuestas de acción se orientan a la generación de políticas que reduzcan o 
eliminen los factores individuales y sociales modificables que se consideran evitables o injustos como el nivel educativo, los ingresos, los riesgos laborales y el acceso a servicios sanitarios (18). Por su parte, la DSSE plantea que existe una subsunción de lo biológico en lo social entendido este como una realidad histórica en donde existen asimetrías en las relaciones de poder y dominación, particularmente de clase social, género y etnia, que deben ser visibilizadas para comprender la configuración de los modos de vida que generan vulnerabilidades diferenciales en salud tanto a nivel individual como a nivel colectivo y así plantear propuestas transformadoras en donde los sujetos y grupos sociales que han resultado "enajenados de sus posibilidades de realización vital" asuman una participación política más activa que conlleve a reconfigurar las relaciones de poder para construir proceso de emancipación que permitan superar las inequidades sociales reduciendo así las desigualdades sanitarias $(19,20)$. En este orden de ideas, el enfoque más funcional a la estructura institucional es el de los DSS; si los resultados del ENSAB IV, hubiesen planteado las apuestas praxiológicas derivadas del marco teórico de la DSSE propuesta en el protocolo, las prácticas institucionales caracterizadas por privilegiar acciones pragmáticas para resolver las causas inmediatas de la enfermedad, seguramente resultarían inconsecuentes generando fisuras entre la evidencia científica producida por el mismo Estado y sus formas de actuar frente a los problemas sanitarios, en este caso de salud bucal. Por tanto, puede leerse entre líneas, un interés tácito de los tomadores de decisiones de no ahondar en aspectos estructurales ni visibilizarlos, ya que les implicaría impulsar acciones que conlleven a transformaciones profundas en la configuración de las relaciones de poder, algo casi impensable en una institución caracterizada por unas formas de hacer prestablecidas influenciadas por un contexto político, sanitario, económico e histórico (2,21). 
En cuanto a lo metodológico el ENSAB IV, permite evidenciar una desarticulación entre los componentes cuantitativo y cualitativo, esta tensión va más allá de una dificultad de operacionalizar una triangulación de técnicas, tiene que ver con los paradigmas que los soportan. Por un lado, la metodología cuantitativa en salud se enmarca en el positivismo biomédico que ofrece una representación matemática del mundo, mientras que por otro lado, la metodología cualitativa enmarcada en el paradigma hermenéutico o interpretativo, intenta comprender la realidad social con su contexto y visibilizar el significado humano atribuido a los fenómenos sociales (22). Aunque en el protocolo del ENSAB IV se planteó una articulación de estas metodologías para lograr una aproximación más holística a la situación de salud bucal del país; en la ejecución del estudio se evidencio una subordinación del componente cualitativo y una hegemonía del componente cuantitativo evidenciando que sigue primando la reducción de la realidad al plano empírico en donde la expresión biológica de la enfermedad sigue siendo el punto de partida y los principales intereses de estudio consisten en hallar explicaciones causales a-históricas o factores de riesgo bio-psíquicos, comportamentales, medioambientales, sociales como si el estudio del ser humano pudiera desligarse de la realidad social que lo determina, invisibilizando la relación dialéctica hombre-sociedad $(23,24)$.

\section{CONCLUSIONES}

El ENSAB IV fue un ejercicio investigativo que planteo importantes retos para los estudios nacionales en salud, ya que, al asumir una postura epistemológica alternativa, dejó en evidencia las tensiones y hegemonías que existen tanto en las ciencias que sustentan el conocimiento en salud como en los intereses políticos de distintos actores. Dichas tensiones explican en gran parte, los 
avances y limitaciones que han tenido las intervenciones sanitarias realizadas por el Estado y los desafíos que enfrenta para garantizar el derecho fundamental a la salud y lograr mayores niveles de equidad.

El tener un enfoque teórico metodológico crítico y dos instituciones con equipos de trabajo diferentes uno encargado de la fase de diseño y otro en la ejecución del ENSAB IV, favoreció que se evidenciaran una serie de tensiones y discontinuidades como se evidencia tanto en los documentos publicados como en las percepciones de los actores participantes en el estudio. Las principales tensiones evidenciadas fueron: la apropiación y operacionalización del enfoque teórico metodológico propuesto en el diseño del estudio; institucional para mostrar resultados desde posturas y enfoques críticos; en la articulación del componente cualitativo del estudio; tener dos instituciones con actores diferentes en el diseño y ejecución del estudio.

Para terminar, se podría afirmar que, aunque los textos de metodología de la investigación indiquen que el protocolo debe ser elaborado de tal manera que cualquier investigador pueda desarrollarlo, sin embargo, el ENSAB IV es un claro ejemplo de los inconvenientes, discontinuidades, tensiones y desafíos que se generan cuando una institución realiza el diseño y otra diferente realiza la ejecución de ese protocolo como lo refieren varios de los actores participantes en este estudio.

\section{AGRADECIMIENTOS}

Por el apoyo financiero al Departamento Administrativo de Ciencia Tecnología e Innovación (COLCIENCIAS - Universidad Nacional de Colombia contrato de financiamiento de reembolso 
condicional NFP44842-139-2017), convocatoria Doctorados Nacionales 727 de 2015 - código HERMES (39867).

\section{REFERENCIAS}

1. Universidad de Antioquia - Facultad Nacional de Salud Pública. Cuarto Estudio Nacional de Salud Bucal- Protocolo del Estudio. Medellín, Colombia: Universidad de Antioquia; 2009.

2. Pardo Romero F, Hernandez L. Enfermedad periodontal: enfoques epidemiológicos para su análisis como problema de salud pública. Rev salud pública [Internet]. 2018; 20(2): 258-64. Available from: http://www.scielo.org.co/pdf/rsap/v20n2/0124-0064-rsap-20-02-258.pdf

3. República de Colombia. Ministerio de salud y Protección Social. Cuarto Estudio Nacional de Salud Bucal - ENSAB IV. Situación en Salud Bucal. Bogotá; 2015.

4. Ministerio de la Protección Social. Decreto 3039 de 2007. "Por el cual se adopta el Plan Nacional de Salud Pública 2007-2010”. [Internet]. Bogotá.; 2007 p. 1-54. Available from: http://santafedeantioquia-antioquia.gov.co/apc-aa-files/pdf.

5. República de Colombia. Corte Constitucional. Sentencia No T-760 de 2008. Bogotá, D.C; 2008.

6. Heredia N, Laurell AC, Feo O, Noronha J, González-Guzmán R, Torres-Tovar M. The right to health: what model for Latin America? Lancet (London, England) [Internet]. 2015; 385(9975): e34-7. Available from: http://www.sciencedirect.com/science/article/pii/S0140673614614938

7. Morales-borrero C, Borde E. ¿Determinación social o determinantes sociales? Diferencias 
conceptuales e implicaciones praxiológicas. Fac Nac Salud Pública [Internet]. 2013; 15(6): 797-808. Available from: http://www.scielosp.org/pdf/rsap/v15n6/v15n6a03.pdf

8. World Health Organization. ORAL HEALTH SURVEYS -BASIC METHODS. Geneve; 1997.

9. Ministerio de salud y Protección social. RESOLUCION 1841 DE 2013 - Plan Decenal de Salud Publica 2012-21. 2013.

10. Ministerio de Salud República de Colombia. Resolución N 008430. 1993;1993:12. Available from:http://www.unisabana.edu.co/fileadmin/Documentos/Investigacion/comite_de_etica/Re s_8430_1993_-_Salud.pdf

11. Ministerio de Salud y proteccion social. Cuarto Estudio Nacional de Salud Bucal (ENSAB IV). Metodología y Determinación Social de la Salud Bucal. Bogotá, D.C; 2015.

12. Organización Mundial de la Salud (OMS). Cerrando la brecha: la política de acción sobre los determinantes sociales de las salud. Conferencia Mundial sobre los Determinantes Sociales de la Salud [Internet]. Río de Janeiro.; 2011. Available from: http://www.paho.org/SaludenlasAmericas/index.php

13. Organización Mundial de la Salud. ¿Que es la salud? onible en: http://www.who.int/suggestions/faq/es/. 2014.

14. Laurell AC. La salud enfermedad como proceso social. Rev Latinoam Salud. 1981; 2(1): 725.

15. Iriart C, Waitzkin H, Breilh J, Estrada A, Merhy EE. Medicina social latinoamericana: aportes y desafíos. Rev Panam Salud Pública. 2002; 12(2): 128-36.

16. Colectiva S. La Medicina Social Latinoamericana y de Equidad en Salud. 2012;

17. Breilh J. La determinación social de la salud como herramienta de transformación hacia una 
nueva salud pública ( salud colectiva ). Rev Fac Nac Salud Pública. 2013; 31(1): 13-27.

18. Diderichsen F, Evans T, Whitehead M. El desafío de la falta de equidad en Salud. De la ética a la acción. 2002. 13-25 p.

19. Breilh J. Las tres " s " de la determinación de la vida y el triángulo de la política. Cent Bras Estud saúde. 2012;19-20.

20. Breilh J. Epidemiología Crítica - Ciencia Emancipadora e Interculturalidad. 2003. Capitulo IV y VII: Pags 137-60; 197-224.

21. Molina G, Ramírez A, Ruiz A. Tensiones en las decisiones en Salud Pública en el sistema de salud colombiano: El bién común en confrontación con los intereses y prácticas particulares. Medellín: Universidad de Antioquía; 2014. 504 p.

22. De la Cuesta C. Investigación en salud: reflexiones en torno a los trabajos de Testa y Navarro. Rev Fac Nac Salud Pública. 1996; 13(2): 51-62.

23. Breilh J. Una perspectiva emancipadora de la investigación e incidencia basada en la determinación social de la salud. ¿Determinación Social o Determinantes Sociales de la salud? Memorias del Taller Latinoamericano sobre Determinación Social de la Salud. 2011; 290.

24. Martínez G. Los saberes que han sido subordinados en la salud. Rev CONAMED. 2015; 20 (1): $32-7$. 